\title{
Obesity and Risk of Breast Cancer Mortality in Hispanic and Non-Hispanic White Women: The New Mexico Women's Health Study
}

\author{
Avonne E. Connor, PhD, Richard N. Baumgartner, PhD, Christina Pinkston, MS, and Kathy B. Baumgartner, PhD
}

\begin{abstract}
Obesity is reported to be associated with poorer survival in women with breast cancer, regardless of menopausal status. Our purpose was to determine if the associations of obesity with breast cancer-specific, all-cause, and non-breast cancer mortality differ between Hispanic and non-Hispanic white (NHW) women with breast cancer. Data on lifestyle and medical history were collected for incident primary breast cancer cases (298 NHW, 279 Hispanic) in the New Mexico Women's Health Study. Mortality was ascertained through the National Death Index and New Mexico Tumor Registry over 13 years of follow-up. Adjusted Cox regression models indicated a trend towards increased risk for breast cancer-specific mortality in obese NHW women (hazard ratio [HR] 2.07; 95\% confidence interval [CI] 0.98-4.35) but not in Hispanic women (HR 1.32; 95\% CI 0.64-2.74). Obese NHW women had a statistically significant increased risk for all-cause mortality (HR 2.12; 95\% CI 1.15-3.90) while Hispanic women did not (HR 1.23; 95\% CI 0.71-2.12). Results were similar for non-breast cancer mortality: NHW (HR 2.65; 95\% CI 0.90-7.81); Hispanic (HR 2.18; 95\% CI 0.77-6.10). Our results suggest that obesity is associated with increased risk for breast cancer-specific mortality in NHW women; however, this association is attenuated in Hispanic women.
\end{abstract}

\section{Introduction}

$\mathrm{O}$ BESITY IS ASSOCIATED WITH SEVERAL chronic health conditions. $^{1-3}$ It is reported to be a significant risk factor for breast cancer in postmenopausal women, although either no association or a reduced risk has been reported in premenopausal women. ${ }^{2,4-6}$ Obesity around the time of diagnosis also has been reported to be associated with poorer survival and increased likelihood of recurrence in women with breast cancer, regardless of menopausal status, stage of disease, or treatment. ${ }^{2,7}$ Women with breast cancer may gain weight following treatment, which influences their quality of life. ${ }^{8,9}$ An estimated 11,000 to 18,000 breast cancer deaths per year in U.S. women $>50$ years of age could be prevented if women maintained a body mass index (BMI) of $<25 \mathrm{~kg} / \mathrm{m}^{2}$ throughout their adult lives. ${ }^{10}$ The association of obesity with breast cancer has been attributed to increased concentrations of free estradiol and free testosterone in obese postmenopausal women. ${ }^{2,5}$

Several recent prospective studies have examined the relationship between obesity and breast cancer-specific mortality and reported relative risks ranging from 1.45 to $1.57 .5,11,12$ The purpose of the present analyses was to deter- mine if obesity (BMI $>30 \mathrm{~kg} / \mathrm{m}^{2}$ ) is associated with breast cancer-specific, all-cause, and non-breast cancer mortality, and if these associations differ by Hispanic versus nonHispanic white (NHW) ethnicity based on data for participants in the New Mexico Women's Health Study (NMWHS).

\section{Materials and Methods}

The NMWHS was a statewide population-based casecontrol study of breast cancer in Hispanic and NHW women described previously in detail. ${ }^{13-16}$ Women diagnosed with a new primary breast cancer were ascertained through the New Mexico Tumor Registry (NMTR), a member of the Surveillance, Epidemiology and End Results (SEER) Program of the National Cancer Institute. Women diagnosed with invasive or in situ breast carcinoma from January 1, 1992, through December 31, 1994, who were residents of the state and between 20 and 80 years of age at diagnosis, were eligible for the study.

\section{Identification of study subjects}

All Hispanic cases $(n=491)$ ascertained and approximately $33 \%$ of NHW cases $(n=493)$, selected using a stratified 
random selection process, were eligible for participation. Sampling fractions were based on the ratio of Hispanic to NHW cases by age group and geographic location (planning district) using data from a previous 3-year period (1988-1990). Population-based controls were selected using a modified random-digit dialing method and were frequency matched to cases based on ethnicity, age group (30-39, 40-64, 65-74 years), and the seven health planning districts. Of the 984 eligible women identified, 73\% completed an interview: 339 Hispanic and 383 NHW. Specific details on nonparticipation were reported previously. ${ }^{13}$

\section{Exclusions}

Case ascertainment for the NMWHS was restricted to a first primary breast cancer diagnosis. It was subsequently determined at the time of follow-up study (2007-2011) that 29 cases had a prior breast cancer diagnosis due to a delay in data abstraction for cases diagnosed in outlying areas of the state or outside of the state. These subjects were excluded from survival analyses. Another case was excluded because the subject did not have a confirmed breast cancer diagnosis but rather another cancer at study enrollment. Five other cases were excluded due to missing breast cancer stage at diagnosis. Lastly, 110 in situ cases were excluded, resulting in a final sample of 577 invasive cases for survival analyses (Fig. 1). One additional case was excluded from breast cancer-specific and non-breast cancer mortality analyses because the primary cause of death was unknown.

\section{Data collection-anthropometric measurement and covariates}

A lifestyle questionnaire administered by trained interviewers was used to collect data on demographic characteristics, reproductive and medical histories, oral contraceptive and hormone use, family history of cancer, alcohol and cigarette smoking history, height, current weight, usual adult weight, weight at age 18, physical activity, and breast cancer surgery and treatment. Dietary data were collected using a validated 140-item food frequency questionnaire (FFQ) and used to estimate usual nutrient intake. ${ }^{17,18}$ This FFQ incorporated unique food items specific to New Mexico women. The referent period for food intake was approximately 6 months prior to the diagnosis date for cases or interview date for controls.

Subjects reported their height in feet and inches and current weight and at age 18 in pounds. Body mass index (BMI) was calculated with weight in kilograms and height in meters and was categorized as $\leq 24.9 \mathrm{~kg} / \mathrm{m}^{2}$ (normal), $25.0-29.9 \mathrm{~kg} / \mathrm{m}^{2}$ (overweight), and $\geq 30 \mathrm{~kg} / \mathrm{m}^{2}$ (obese). ${ }^{19}$ BMI at age 18 was categorized as $<18.5 \mathrm{~kg} / \mathrm{m}^{2}$ (underweight), $18.5-24.9 \mathrm{~kg} / \mathrm{m}^{2}$ (normal weight), $25.0-29.9 \mathrm{~kg} / \mathrm{m}^{2}$ (overweight), and $\geq 30 \mathrm{~kg} /$ $\mathrm{m}^{2}$ (obese). ${ }^{19}$ Education in years was categorized as $<12,12$,

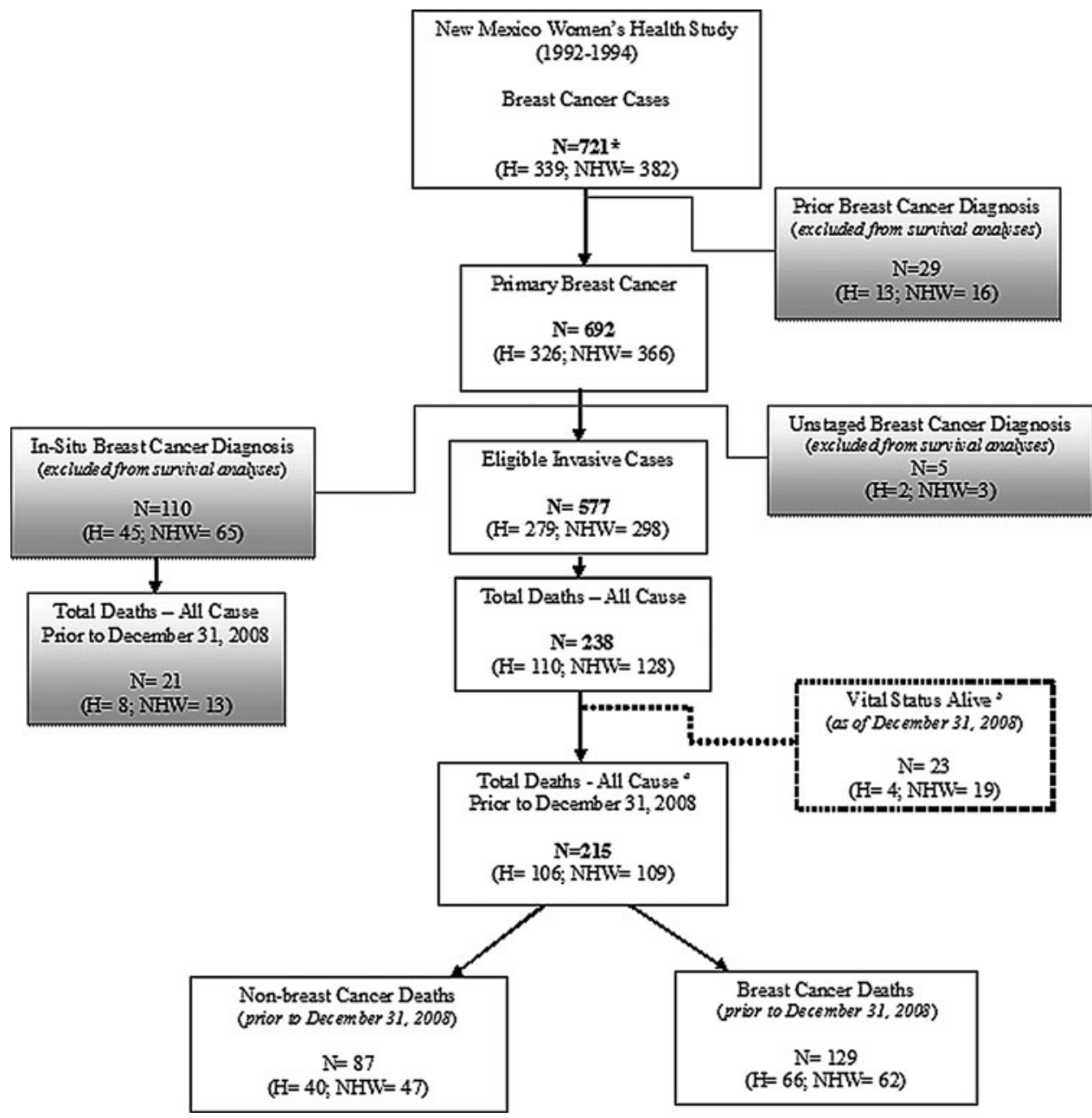

FIG. 1. Distribution of cases by vital status and inclusion criteria for survival analysis. NDI, National Death Index; NMTR, New Mexico Tumor Registry; H, Hispanic; NHW, non-Hispanic white. *One case was excluded for not having a confirmed breast cancer diagnosis, but some other form of cancer at study enrollment. ${ }^{\mathrm{a}}$ Deaths ascertained from NDI $(n=212$; $\mathrm{H}=106$; $\mathrm{NHW}=106)$ and NMTR $(n=3 ; \mathrm{H}=0 ; \mathrm{NHW}=$ $3) .{ }^{b}$ Deaths ascertained from NMTR only $(n=23 ; \mathrm{H}=4$; $\mathrm{NHW}=19)$. 
or $>12$ years. Parity was categorized as nulliparous, 1, 2, 3, or $\geq 4$. Family history of breast cancer was based upon breast cancer diagnosis in mother, sister, or daughter. Comorbidity included self-reported history (yes, no) of diabetes, high cholesterol, high blood pressure, stroke, and heart attack. Age at diagnosis, duration of estrogen hormonal therapy in years, and energy intake of calories per day were included as continuous variables. Smoking history was coded as never, former, or current. Physical activity was calculated as the total metabolic equivalent task (METs) score in hours per week. ${ }^{20}$ Menopausal status was categorized as premenopausal, postmenopausal, or surgical/unknown based on self-reported menstrual history, history of hysterectomy (with or without oophorectomy), and use of hormone replacement therapy ${ }^{13}$.

Breast cancer prognostic markers and treatment information were abstracted from hospital records by NMTR. Estrogen receptor (ER) status was coded as ER positive (ER+), ER negative (ER-), or unknown. Cross-classification by progesterone receptor (PR) status was not considered in the present analyses due to the small numbers of discordant pairs (i.e., $\mathrm{ER}+/ \mathrm{PR}-$ or $\mathrm{ER}-/ \mathrm{PR}+)$. Breast cancer stage was categorized as local versus regional and distant. Breast cancer treatment was categorized as no surgery, surgery only, surgery plus radiation, surgery plus chemotherapy, and surgery plus radiation and any chemotherapy.

Written informed consent was obtained at the time of the interview. The NMWHS baseline and follow-up studies were approved and monitored by the Institutional Review Board committees at the University of New Mexico and University of Louisville.

\section{Outcome assessment}

Deaths were ascertained through the National Death Index (NDI), as well as NMTR, Lexus Nexus Accurint website (http://www.accurint.com), the Social Security Death Index (http://ssdi.rootsweb.ancestry.com), online obituaries, family notifications, and returned mail. Mortality data from NDI were collected through December 31, 2008, the most recent date for which NDI data were available. For cases, survival data were merged with updated data for clinical and tumor characteristics from NMTR.

The majority (99\%) of deaths due to any cause were accounted for by NDI submissions. The following variables were submitted to NDI for death identification purposes: last name, first name, middle initial, social security number, birth date, father's surname, age at death, race, sex, state of residence, and state of birth. Matching variables were then created based on the variables submitted to verify NDI results for subjects. A total of 135 subjects were considered NDI "perfect matches" because the majority of important variables submitted matched with data collected for NDI records (last name, first name, middle initial, social security number, birth date). A visual inspection of NDI record results was made in order to classify the remaining subjects. A total of 99 subjects were classified as "partial matches" due to missing or incorrect information for at least one identifying variable submitted to NDI. There were 458 subjects remaining who did not match on the variables included in the NDI submissions. An additional three deaths due to any cause were also identified from NMTR results.

\section{Statistical analyses}

The outcomes for the present analyses were breast cancerspecific mortality, all-cause mortality due to any cause, and non-breast cancer mortality. Breast cancer-specific mortality was identified as ICD codes C50, malignant neoplasm of breast of unspecified site, or 174, malignant neoplasm of female breast, for primary cause of death on the death certificate. Death due to any cause was defined as a death occurring during the selected time period, including breast cancer deaths. The follow-up period was time from the date of diagnosis to death date, or December 31, 2008, if the subject was still alive.

Characteristics of the cases at baseline were compared across the BMI subgroups and by ethnicity using the chisquare statistic for categorical variables and ANOVA for continuous variables. Kaplan-Meier survival analysis was used to compare groups for breast cancer-specific, all-cause, and non-breast cancer mortality. Log-rank $p$ values were calculated to test for significant differences between ethnicity and BMI categories. Multivariable Cox proportional hazards regression was used to estimate hazard ratios (HRs) and 95\% confidence intervals (CIs) for the association of BMI with breast cancer-specific, all-cause, and non-breast cancer mortality. ${ }^{21}$ Univariable analyses were performed to screen for potential confounders using a $p$ value of 0.20 . Stratified analyses were examined for effect modification by ethnicity and menopausal status for all-cause and non-breast cancer mortality and by ER status for breast cancer-specific mortality. Covariates were considered confounders if they produced a change in the point estimate for BMI of $\geq 10 \%$. $^{22}$ Tests of trend were computed using the median values within each category of BMI. All analyses were performed using the Statistical Analysis System (SAS) software, version 9.2 (SAS Institute Inc., Cary, NC).

\section{Results}

Mean follow-up time was approximately 13 years. There were a total of 215 deaths (106 Hispanic, 109 NHW), and 129 of these invasive cases were considered breast cancer-specific deaths (Fig. 1).

Baseline characteristics stratified by BMI at interview and ethnicity are shown in Table 1 . Twenty-eight percent of Hispanics $(n=77)$ compared to $16 \%$ of NHW $(n=47)$ women were obese. Among the women who were overweight at age 18, more Hispanics were obese at time of interview (16\%) compared with NHW (2\%). Approximately 27\% Hispanic and $11 \%$ NHW women who were obese had a history of Type 2 diabetes. Hispanic women consumed more calories per day, regardless of BMI level. There were significant differences $(p<0.05)$ between BMI and ethnic groups for parity, history of diabetes, education, and menopausal status. The average age at diagnosis was 53.2 years $(\mathrm{SD}=11.2)$.

Figures 2, 3, and 4 show Kaplan-Meier survival curves for breast cancer-specific mortality, all-cause mortality, and nonbreast cancer mortality by ethnicity and BMI. Differences between curves were observed only for all-cause mortality (log-rank $p=0.06)$. We tested the proportional hazards assumption by modeling interaction terms of time and BMI and found no significant interactions.

Obesity at time of interview was significantly associated with all-cause mortality $\left(\mathrm{HR}=1.50 ; 95 \% \mathrm{CI} 1.03-2.17, p_{\text {trend }}=\right.$ 0.05 ) and nonsignificantly with increased risk of breast 
Table 1. Baseline Characteristics for Women Diagnosed with a First New Primary Breast Cancer,

New Mexico Women's Health Study, 1992-1994

\begin{tabular}{|c|c|c|c|c|c|c|c|c|c|c|c|c|c|c|c|}
\hline \multirow[b]{3}{*}{ Characteristics } & \multicolumn{14}{|c|}{ BMI category at interview } & \multirow[b]{3}{*}{$\operatorname{p}_{\text {value }}^{\mathrm{t}}$} \\
\hline & \multicolumn{8}{|c|}{ Hispanic $(\mathrm{N}=279)$} & \multicolumn{6}{|c|}{ Non-Hispanic white $(\mathrm{N}=298)$} & \\
\hline & \multicolumn{2}{|c|}{$\begin{array}{l}\leq 24.9 \\
\mathrm{~N}=105\end{array}$} & \multicolumn{2}{|c|}{$\begin{array}{c}25-29.9 \\
\mathrm{~N}=97\end{array}$} & \multicolumn{2}{|c|}{$\begin{array}{c}\geq 30 \\
\mathrm{~N}=77\end{array}$} & \multirow[t]{2}{*}{ value $^{\mathrm{p}}$} & \multicolumn{2}{|c|}{$\begin{array}{l}\leq 24.9 \\
\mathrm{~N}=166\end{array}$} & \multicolumn{2}{|c|}{$\begin{array}{l}25-29.9 \\
\mathrm{~N}=85\end{array}$} & \multicolumn{2}{|c|}{$\begin{array}{l}\geq 30 \\
\mathrm{~N}=47\end{array}$} & $\underset{\text { value }^{\mathrm{a}}}{\mathrm{p}}$ & \\
\hline & $\mathrm{N}$ & $\%$ & \multicolumn{3}{|c|}{ BMI, age $18\left(\mathrm{~kg} / \mathrm{m}^{2}\right)$} & $\%$ & & $\mathrm{~N}$ & $\%$ & $\mathrm{~N}$ & $\%$ & $\mathrm{~N}$ & $\%$ & & \\
\hline$<18.5$ & 26 & 26.0 & 17 & 18.1 & 7 & 9.2 & $<0.01$ & 42 & 25.3 & 16 & 19.3 & 5 & 10.6 & $<0.01$ & 0.28 \\
\hline $18.5-24.9$ & 74 & 74.0 & 74 & 78.7 & 55 & 72.4 & & 120 & 72.3 & 64 & 77.1 & 38 & 80.9 & & \\
\hline $25-29.9$ & 0 & 0 & 3 & 3.2 & 12 & 15.8 & & 3 & 1.8 & 2 & 2.4 & 1 & 2.1 & & \\
\hline$\geq 30$ & 0 & 0 & 0 & 0 & 2 & 2.6 & & 1 & 0.6 & 1 & 1.2 & 3 & 6.4 & & \\
\hline \multicolumn{16}{|l|}{ Education (years) } \\
\hline$<12$ & 45 & 43.3 & 35 & 36.1 & 33 & 42.9 & 0.28 & 36 & 21.8 & 26 & 30.6 & 17 & 36.2 & 0.06 & $<0.01$ \\
\hline 12 & 26 & 25.0 & 32 & 33.0 & 28 & 36.4 & & 12 & 7.3 & 2 & 2.4 & 3 & 6.4 & & \\
\hline$>12$ & 33 & 31.7 & 30 & 30.9 & 16 & 20.8 & & 117 & 70.9 & 57 & 67.1 & 27 & 57.5 & & \\
\hline Parity & & & & & & & & & & & & & & & \\
\hline Nulliparous & 17 & 16.2 & 6 & 6.2 & 10 & 13.0 & 0.02 & 37 & 23.3 & 6 & 7.1 & 4 & 8.5 & $<0.01$ & $<0.01$ \\
\hline 1 & 16 & 15.2 & 2 & 2.1 & 5 & 6.5 & & 33 & 19.9 & 12 & 14.1 & 5 & 10.6 & & \\
\hline 2 & 23 & 21.9 & 16 & 16.5 & 15 & 19.5 & & 48 & 28.9 & 29 & 34.1 & 19 & 40.4 & & \\
\hline 3 & 19 & 18.1 & 35 & 36.1 & 15 & 19.5 & & 31 & 18.7 & 14 & 16.5 & 8 & 17.0 & & \\
\hline$\geq 4$ & 30 & 28.6 & 38 & 39.2 & 32 & 41.6 & & 17 & 10.2 & 24 & 28.2 & 11 & 23.4 & & \\
\hline Family history & & & & & & & & & & & & & & & \\
\hline Yes & 11 & 10.5 & 10 & 10.3 & 11 & 14.3 & 0.45 & 21 & 12.7 & 15 & 17.7 & 6 & 12.8 & 0.69 & 0.35 \\
\hline No & 94 & 89.5 & 87 & 89.7 & 66 & 85.7 & & 145 & 87.4 & 70 & 82.4 & 41 & 87.2 & & \\
\hline History of diabetes & & & & & & & & & & & & & & & \\
\hline Yes & 8 & 7.6 & 11 & 11.3 & 21 & 27.3 & $<0.01$ & 2 & 1.2 & 4 & 4.7 & 5 & 10.6 & $<0.01$ & $<0.01$ \\
\hline No & 97 & 92.4 & 86 & 88.7 & 56 & 72.7 & & 164 & 98.8 & 81 & 95.3 & 42 & 89.4 & & \\
\hline History of high cholester & & & & & & & & & & & & & & & \\
\hline Yes & 25 & 23.8 & 20 & 20.6 & 20 & 26.0 & 0.79 & 34 & 20.5 & 34 & 40.0 & 14 & 29.8 & 0.03 & 0.25 \\
\hline No & 80 & 76.2 & 77 & 79.4 & 57 & 74.0 & & 132 & 79.5 & 51 & 60.0 & 33 & 70.2 & & \\
\hline History of high blood pre & essure & & & & & & & & & & & & & & \\
\hline Yes & 22 & 21.0 & 30 & 30.9 & 32 & 41.6 & $<0.01$ & 40 & 24.1 & 31 & 36.5 & 23 & 48.9 & $<0.01$ & 0.71 \\
\hline No & 83 & 79.1 & 67 & 69.1 & 45 & 58.4 & & 126 & 75.9 & 54 & 63.5 & 24 & 51.1 & & \\
\hline History of stroke & & & & & & & & & & & & & & & \\
\hline Yes & 3 & 2.9 & 3 & 3.1 & 0 & 0 & 0.22 & 1 & 0.6 & 1 & 1.2 & 2 & 4.3 & 0.08 & 0.45 \\
\hline No & 102 & 97.1 & 94 & 96.9 & 77 & 100 & & 165 & 99.4 & 84 & 98.8 & 45 & 95.7 & & \\
\hline History of heart disease & & & & & & & & & & & & & & & \\
\hline Yes & 4 & 3.8 & 2 & 2.1 & 3 & 3.9 & 0.97 & 9 & 5.4 & 5 & 5.9 & 3 & 6.4 & 0.79 & 0.15 \\
\hline No & 101 & 69.2 & 95 & 97.9 & 74 & 96.1 & & 157 & 94.6 & 80 & 94.1 & 44 & 93.6 & & \\
\hline Estrogen receptor status & & & & & & & & & & & & & & & \\
\hline $\mathrm{ER}+$ & 64 & 64.7 & 54 & 60.7 & 49 & 71.0 & 0.46 & 104 & 69.8 & 58 & 77.3 & 27 & 69.2 & 0.70 & 0.09 \\
\hline ER- & 35 & 35.4 & 35 & 39.3 & 20 & 29.0 & & 45 & 30.2 & 17 & 22.7 & 12 & 30.8 & & \\
\hline Stage of breast cancer & & & & & & & & & & & & & & & \\
\hline Local & 61 & 58.1 & 58 & 59.8 & 42 & 54.6 & 0.86 & 110 & 66.3 & 56 & 65.9 & 30 & 63.8 & 0.50 & 0.05 \\
\hline Regional & 39 & 37.1 & 37 & 38.1 & 34 & 44.2 & & 54 & 32.5 & 27 & 31.8 & 13 & 27.7 & & \\
\hline Distant & 5 & 4.8 & 2 & 2.1 & 1 & 1.3 & & 2 & 1.2 & 2 & 2.4 & 4 & 8.5 & & \\
\hline Menopausal status & & & & & & & & & & & & & & & \\
\hline Premenopausal & 52 & 49.5 & 36 & 37.5 & 26 & 34.2 & 0.03 & 57 & 34.3 & 17 & 20.0 & 13 & 27.7 & 0.25 & 0.01 \\
\hline Postmenopausal & 49 & 46.7 & 53 & 55.2 & 45 & 59.2 & & 97 & 58.4 & 64 & 75.3 & 31 & 66.0 & & \\
\hline Surgical/unknown & 4 & 3.8 & 7 & 7.3 & 5 & 6.6 & & 12 & 7.2 & 4 & 4.7 & 3 & 6.4 & & \\
\hline Breast cancer treatment & & & & & & & & & & & & & & & \\
\hline No su & 1 & 1.0 & 1 & 1.0 & 0 & 0 & 0.84 & 2 & 1.2 & 1 & 1.8 & 2 & 4.3 & 0.88 & 0.66 \\
\hline Surget & 37 & 35.2 & 29 & 29.9 & 29 & 37.7 & & 59 & 35.5 & 30 & 35.3 & 16 & 34.0 & & \\
\hline Surgery and radiation & 22 & 21.0 & 22 & 22.7 & 13 & 16.9 & & 38 & 22.9 & 19 & 22.4 & 9 & 19.2 & & \\
\hline $\begin{array}{l}\text { Surgery and } \\
\text { chemotherapy }\end{array}$ & 16 & 15.2 & 23 & 23.7 & 15 & 19.5 & & 26 & 15.7 & 14 & 16.5 & 13 & 27.7 & & \\
\hline $\begin{array}{l}\text { Surgery, radiation, } \\
\text { and chemotherapy }\end{array}$ & 29 & 27.6 & 22 & 22.7 & 20 & 26.0 & & 41 & 24.7 & 21 & 24.7 & 7 & 14.9 & & \\
\hline
\end{tabular}


TABle 1. (CONTINUED)

\begin{tabular}{|c|c|c|c|c|c|c|c|c|c|c|c|c|c|c|c|}
\hline \multirow[b]{3}{*}{ Characteristics } & \multicolumn{14}{|c|}{ BMI category at interview } & \\
\hline & \multicolumn{8}{|c|}{ Hispanic $(\mathrm{N}=279)$} & \multicolumn{6}{|c|}{ Non-Hispanic white $(\mathrm{N}=298)$} & \\
\hline & \multicolumn{2}{|c|}{$\begin{array}{c}\leq 24.9 \\
\mathrm{~N}=105\end{array}$} & \multicolumn{2}{|c|}{$\begin{array}{c}25-29.9 \\
\mathrm{~N}=97\end{array}$} & \multicolumn{2}{|c|}{$\begin{array}{c}\geq 30 \\
N=77\end{array}$} & \multirow[t]{2}{*}{$\underset{\text { value }^{\mathrm{a}}}{\mathrm{p}}$} & \multicolumn{2}{|c|}{$\begin{array}{c}\leq 24.9 \\
\mathrm{~N}=166\end{array}$} & \multicolumn{2}{|c|}{$\begin{array}{c}25-29.9 \\
\mathrm{~N}=85\end{array}$} & \multicolumn{2}{|c|}{$\begin{array}{c}\geq 30 \\
\mathrm{~N}=47\end{array}$} & $\underset{\text { value }^{\mathrm{a}}}{\mathrm{p}}$ & value $^{\mathrm{p}}$ \\
\hline \multicolumn{15}{|l|}{ Smoking history } & \\
\hline Current & 9 & 8.6 & 23 & 23.7 & 10 & 13.0 & 0.04 & 33 & 19.9 & 11 & 12.9 & 4 & 8.5 & 0.19 & 0.25 \\
\hline Former & 26 & 24.8 & 22 & 22.7 & 28 & 36.4 & & 47 & 28.3 & 33 & 38.8 & 16 & 34.0 & & \\
\hline \multirow[t]{2}{*}{ Never } & 70 & 66.7 & 52 & 53.6 & 39 & 50.7 & & 86 & 51.8 & 41 & 48.2 & 27 & 57.5 & & \\
\hline & Mean & $S D$ & Mean & $S D$ & Mean & $S D$ & $\mathrm{value}_{\mathrm{p}}^{\mathrm{p}}$ & Mean & $S D$ & Mean & $S D$ & Mean & $S D$ & value $^{\mathrm{p}}$ & $=$ value $^{\mathrm{p}}$ \\
\hline Age at diagnosis & 51.1 & 12.5 & 51.8 & 13.2 & 52.6 & 11.1 & 0.73 & 53.6 & 11.1 & 57.3 & 8.9 & 53.7 & 10.0 & 0.02 & $2<0.01$ \\
\hline Total METS (h/wk) & 45.0 & 36.2 & 47.3 & 32.7 & 42.6 & 26.8 & 0.65 & 49.1 & 31.7 & 49.4 & 35.7 & 39.8 & 27.2 & 0.19 & 0.36 \\
\hline Calories/day & 2271.5 & 928.1 & 2488.6 & 1141.9 & 2478.2 & 936.5 & 0.24 & 2205.5 & 877.4 & 2181.7 & 759.7 & 2413.9 & 890.7 & 0.27 & 0.03 \\
\hline Estrogen use (years) & 1.4 & 4.5 & 2.0 & 5.1 & 2.4 & 5.4 & 0.40 & 3.8 & 6.9 & 5.4 & 7.3 & 3.7 & 8.0 & 0.20 & $<0.01$ \\
\hline
\end{tabular}

${ }^{\mathrm{a}}$ For tests of differences among women with different body mass index (BMI) status within ethnicity, Mantel-Haenszel $\chi^{2} p$ value for categorical values.

${ }^{\mathrm{b}}$ Ethnic group comparison, regardless of BMI status, Mantel-Haenszel $\chi^{2} p$ value for categorical values.

${ }^{\mathrm{c}}$ For tests of differences among women with different BMI status, ANOVA for continuous variables.

${ }^{\mathrm{d}}$ Ethnic group comparison, regardless of BMI status, $t$-test $p$ value for continuous values.

$\mathrm{ER}+$, estrogen receptor positive; ER - , estrogen receptor negative; METS, metabolic equivalents of tasks.

Missing data: BMI at age $18(n=11)$; education $(n=2)$; menopausal status $(n=2)$; estrogen receptor status $(n=57)$; calories/day $(n=8)$; estrogen use $(n=5)$; total METS $(n=22)$. Note: Percentages may not equal $100 \%$ due to rounding.

cancer-specific death $\left(\mathrm{HR}=1.43 ; 95 \%\right.$ CI $0.89-2.31, p_{\text {trend }}=$ $0.20)$. Associations between obesity and non-breast cancer mortality also were not statistically significant $(\mathrm{HR}=1.60 ; 95 \%$ CI $\left.0.85-3.01, p_{\text {trend }}=0.16\right)$. Covariates considered in multivariable models included breast cancer stage and treatment, energy expenditure (total METs), energy intake (kcals/d), age, menopausal status, smoking history, and comorbid conditions (diabetes, high blood pressure, stroke, coronary heart disease).

Table 2 shows age-adjusted and fully adjusted HRs for breast cancer-specific, all-cause, and non-breast cancer mortality stratified by ethnicity. Although not statistically significant, effect modification by ethnicity was apparent among the age-adjusted models for breast-cancer specific and allcause mortality. After adjusting for BMI at age 18, total METs, duration of estrogen use, breast cancer stage, total daily energy intake, and post-breast cancer diagnosis, breast cancerspecific mortality was positively associated with obesity among NHW women in the fully adjusted model $(\mathrm{HR}=2.07$; $95 \%$ CI 0.98-4.35, $p_{\text {trend }}=0.09$ ); however, this association was substantially attenuated in obese Hispanic women $(\mathrm{HR}=1.32$; $95 \%$ CI $\left.0.64-2.74, p_{\text {trend }}=0.50\right)$. This difference cannot be attributed to loss of statistical power because the number of Hispanic cases $(n=279)$ was only $7 \%$ smaller than the NHW $(n=297)$. Results were similar for all-cause mortality: NHW, $\mathrm{HR}=2.12\left(95 \%\right.$ CI 1.15-3.90, $\left.p_{\text {trend }}=0.03\right)$ versus Hispanic,
FIG. 2. Kaplan-Meier survival curves for breast cancer-specific mortality for each level of body mass index (BMI) by $\mathrm{H}$ and NHW ethnicity.

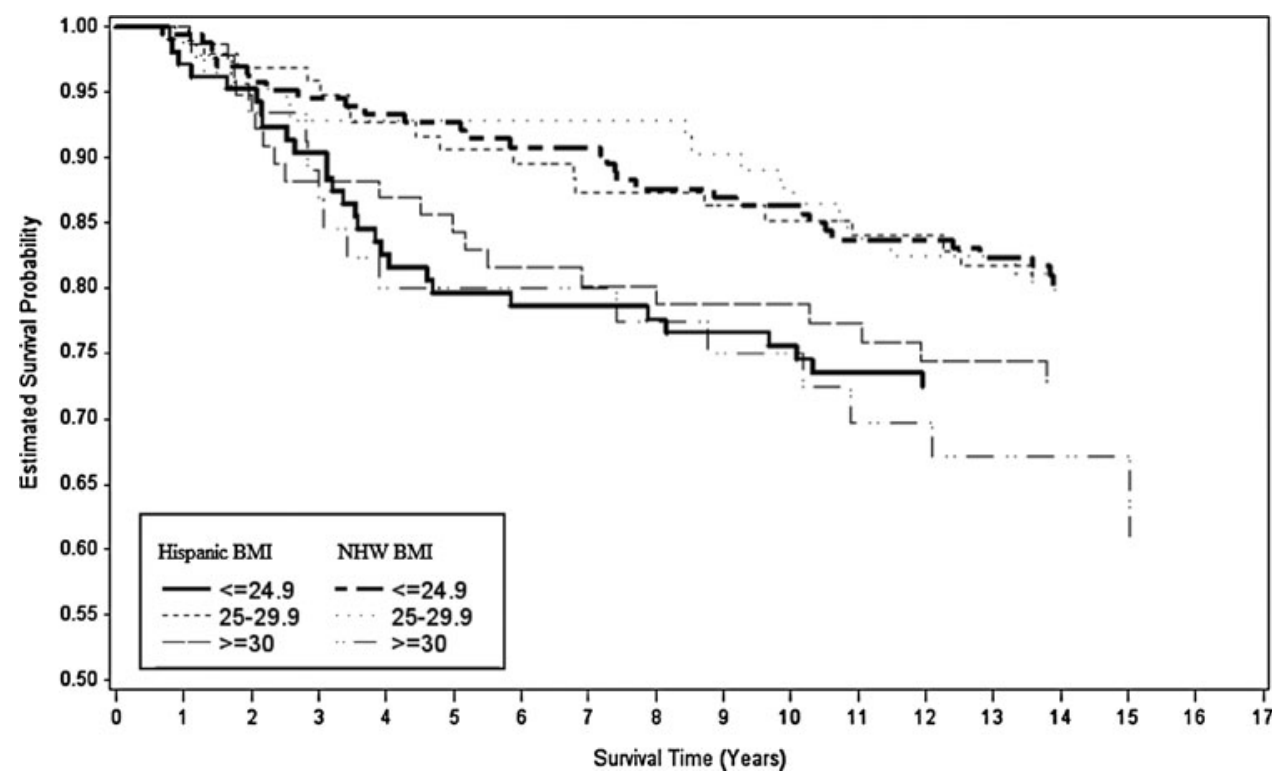




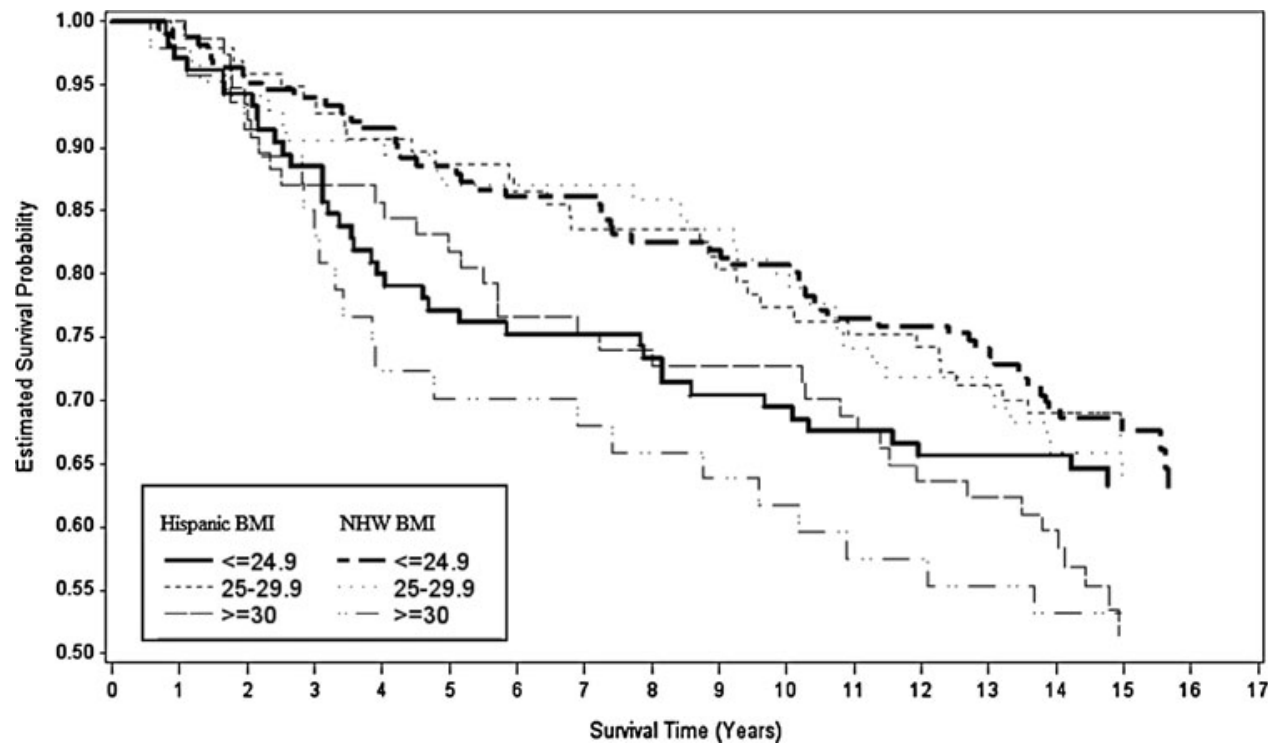

FIG. 3. Kaplan-Meier survival curves for all-cause mortality for each level of BMI by $\mathrm{H}$ and NHW ethnicity.

$\mathrm{HR}=1.23\left(95 \%\right.$ CI 0.71-2.12, $\left.p_{\text {trend }}=0.47\right)$. When non-breast cancer mortality was considered, obese NHW women had a slightly higher risk for death $(\mathrm{HR}=2.65 ; 95 \%$ CI $0.90-7.81$, $\left.p_{\text {trend }}=0.15\right)$ compared with Hispanic women $(\mathrm{HR}=2.18 ; 95 \%$ CI $0.77-6.10, p_{\text {trend }}=0.15$ ) after adjusting for covariates. HRs for all-cause and non-breast cancer mortality were adjusted for age, history of high blood pressure, history of stroke, smoking status, total METs, and total daily energy intake. We also compared the associations between obesity and ethnicity for breast cancer-specific, all-cause, and non-breast cancer mortality by obesity levels measured by BMI $=30-34.9 \mathrm{~kg} / \mathrm{m}^{2}$ and $\mathrm{BMI} \geq 35 \mathrm{~kg} / \mathrm{m}^{2}$; however, results were not significant due to insufficient power. There were only 39 cases (30 Hispanic, $9 \mathrm{NHW}$ ) with BMI levels of $\geq 35 \mathrm{~kg} / \mathrm{m}^{2}$.

Table 3 shows analyses stratified according to ER status. Although results were not statistically significant, the ethnic disparity in risk was found for ER + breast cancer-specific mortality (NHW, HR=2.18 [95\% CI 0.73-6.57, $\left.p_{\text {trend }}=0.23\right]$ versus Hispanics, $\mathrm{HR}=1.58$ [95\% CI $\left.0.63-3.98, p_{\text {trend }}=0.34\right]$ ), while results were similar between ethnic groups for ERbreast cancer-specific mortality, adjusting for BMI at age 18, stage, and treatment.

Stratification by menopausal status was not possible due to the small number of premenopausal invasive cases $(n=201)$. The multiplicative interaction between menopausal status and BMI was not statistically significant in either ethnic group for breast cancer-specific mortality, all-cause mortality, or non-breast cancer mortality (data not shown).

\section{Discussion}

Our results suggest that obesity may be more strongly associated with all-cause and of borderline significance with breast cancer-specific mortality in NHW compared to Hispanic women with breast cancer. In contrast, associations were similar in both ethnic groups for non-breast cancer mortality. This is likely because all-cause mortality includes breast cancer mortality and non-breast cancer mortality. Our

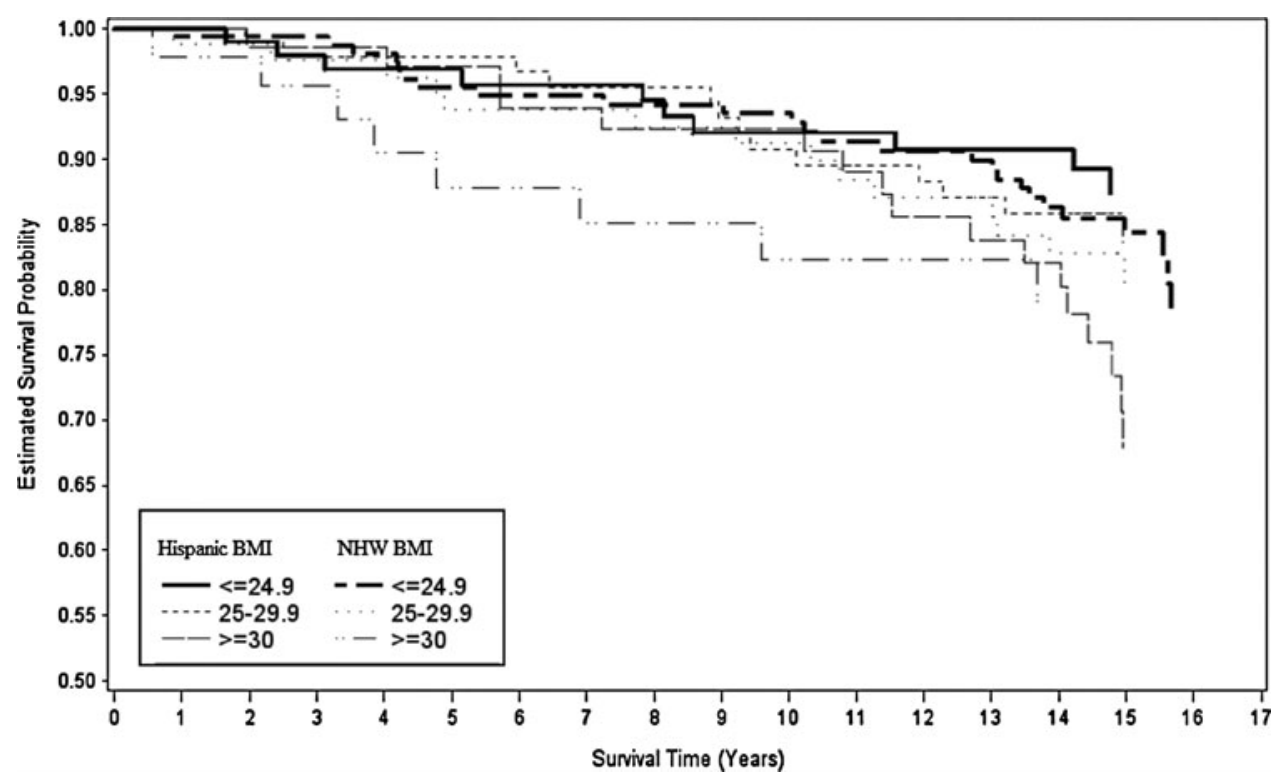

FIG. 4. Kaplan-Meier survival curves for non-breast cancer mortality for each level of BMI by $\mathrm{H}$ and NHW ethnicity. 
Table 2. Comparison of Breast Cancer-Specific Mortality, Non-Breast Cancer, and All-Cause Mortality, Stratified by ETHNicity and Body Mass Index

\begin{tabular}{|c|c|c|c|c|c|c|}
\hline & \multirow[b]{2}{*}{ No. of events } & \multirow[b]{2}{*}{$\mathrm{N}^{a}$} & \multicolumn{3}{|c|}{ BMI $\left(\mathrm{kg} / \mathrm{m}^{2}\right)$ at interview } & \multirow[b]{2}{*}{$\mathrm{p}_{\text {trend }}$} \\
\hline & & & $\begin{array}{c}\leq 24.9 \\
\text { Reference }\end{array}$ & $\begin{array}{c}25-29.9 \\
H R(95 \% C I)\end{array}$ & $\begin{array}{c}\geq 30 \\
\operatorname{HR}(95 \% \mathrm{CI})\end{array}$ & \\
\hline \multicolumn{7}{|l|}{ Mortality, age-adjusted } \\
\hline \multicolumn{7}{|l|}{ Breast cancer-specific } \\
\hline Non-Hispanic white & 62 & 297 & 1.00 & $1.02(0.53,1.94)$ & $1.97(0.99,3.91)$ & 0.09 \\
\hline Hispanic & 66 & 279 & 1.00 & $0.68(0.36,1.27)$ & $0.90(0.47,1.73)$ & 0.72 \\
\hline \multicolumn{7}{|l|}{ All-cause mortality } \\
\hline Non-Hispanic white & 109 & 298 & 1.00 & $0.97(0.60,1.56)$ & $1.79(1.04,3.07)$ & 0.02 \\
\hline Hispanic & 106 & 279 & 1.00 & $0.83(0.51,1.38)$ & $1.26(0.76,2.10)$ & 0.06 \\
\hline \multicolumn{7}{|l|}{ Non-breast cancer } \\
\hline Non-Hispanic white & 47 & 297 & 1.00 & $0.94(0.46,1.89)$ & $1.49(0.58,3.81)$ & 0.04 \\
\hline Hispanic & 40 & 279 & 1.00 & $1.25(0.52,3.00)$ & $2.35(0.99,5.62)$ & 0.53 \\
\hline \multicolumn{7}{|l|}{ Mortality, fully adjusted } \\
\hline \multicolumn{7}{|l|}{ Breast cancer-specific ${ }^{b}$} \\
\hline Non-Hispanic white & 59 & 279 & 1.00 & $0.98(0.49,1.96)$ & $2.07(0.98,4.35)$ & 0.09 \\
\hline Hispanic & 61 & 254 & 1.00 & $0.81(0.41,1.60)$ & $1.32(0.64,2.74)$ & 0.50 \\
\hline \multicolumn{7}{|l|}{ All-cause mortality ${ }^{c}$} \\
\hline Non-Hispanic white & 101 & 284 & 1.00 & $1.02(0.62,1.69)$ & $2.12(1.15,3.90)$ & 0.03 \\
\hline Hispanic & 100 & 265 & 1.00 & $0.87(0.51,1.48)$ & $1.23(0.71,2.12)$ & 0.47 \\
\hline \multicolumn{7}{|l|}{ Non-breast cancer ${ }^{c}$} \\
\hline Non-Hispanic white & 41 & 283 & 1.00 & $1.02(0.46,2.26)$ & $2.65(0.90,7.81)$ & 0.15 \\
\hline Hispanic & 37 & 265 & 1.00 & $1.73(0.63,4.77)$ & $2.18(0.77,6.10)$ & 0.15 \\
\hline
\end{tabular}

${ }^{a}$ Number of cases included in models, one case was excluded from breast cancer-specific and non-breast cancer mortality due to unconfirmed death.

${ }^{\mathrm{b}}$ HRs adjusted for BMI at age 18, total METs, duration of estrogen use, breast cancer stage, total daily energy intake, and post-breast cancer diagnosis.

${ }^{\mathrm{C}}$ HRs adjusted for age, history of high blood pressure, history of stroke, smoking status, total METs, and total daily energy intake.

$\mathrm{HR}$, hazard ratio; CI, confidence interval.

results for NHW women are consistent with reports from meta-analyses $^{23-25}$ and other studies ${ }^{1,3,5,7,10,26}$ that indicate an increased risk for breast cancer mortality in obese compared to nonobese women, with HRs ranging from 1.2 to 3.0. However, few of these previous studies investigated ethnic

Table 3. Risk of Breast Cancer-Specific Mortality for Invasive Breast Cancer Cases by Body Mass Index AND Estrogen Receptor Status

\begin{tabular}{|c|c|c|}
\hline & \multicolumn{2}{|c|}{ Breast cancer-specific mortality ${ }^{\text {a }}$} \\
\hline & $E R+$ & $E R-$ \\
\hline Hispanic & $n=160$ & $n=89$ \\
\hline \multicolumn{3}{|l|}{$\begin{array}{l}\text { BMI }\left(\mathrm{kg} / \mathrm{m}^{2}\right) \\
\text { at interview, HR } \\
(95 \% \mathrm{CI})\end{array}$} \\
\hline$\leq 24.9$ & $1.00(-)$ & $1.00(-)$ \\
\hline $25-29.9$ & $0.73(0.28,1.88)$ & $0.59(0.17,2.02)$ \\
\hline$\geq 30$ & $1.58(0.63,3.98)$ & $0.76(0.18,3.23)$ \\
\hline $\begin{array}{l}p_{\text {trend }} \\
\text { Non-Hispanic white }\end{array}$ & $n=187$ & $\begin{aligned} & 0.66 \\
n= & 74\end{aligned}$ \\
\hline \multicolumn{3}{|l|}{$\begin{array}{l}\mathrm{BMI}\left(\mathrm{kg} / \mathrm{m}^{2}\right) \text { at } \\
\text { interview, HR } \\
(95 \% \mathrm{CI})\end{array}$} \\
\hline$\leq 24.9$ & $1.00(-)$ & $1.00(-)$ \\
\hline $25-29.9$ & $1.10(0.49,2.45)$ & $0.17(0.02,1.35)$ \\
\hline$\geq 30$ & $2.18(0.73,6.57)$ & $0.74(0.11,5.06)$ \\
\hline$p_{\text {trend }}$ & 0.23 & 0.81 \\
\hline
\end{tabular}

${ }^{\mathrm{a}} \mathrm{HRs}$ adjusted for BMI at age 18, stage, and treatment. differences, particularly between Hispanic and NHW women, in the associations of obesity and breast cancer survival. In a recent report from the Multiethnic Cohort Study $(n=3842)$, risk for breast cancer-specific and all-cause mortality was increased in obese women ( $\mathrm{HR}=1.45 ; 95 \%$ CI $1.05-2.00$ and $\mathrm{HR}=1.54 ; 95 \%$ CI 1.23-1.91, respectively), but there was no evidence of an interaction by ethnicity. ${ }^{11}$

In some previous studies, it has been hypothesized that the disparity between Hispanic and NHW women in the associations of obesity with risk and prognosis may be due to different breast cancer phenotypes. ${ }^{27,28}$ Another possible explanation could be ethnic differences in diet. Murtaugh et al. ${ }^{29}$ reported that the Hispanic diet, including more items such as soups, sauces, and vegetables, was inversely associated with risk of breast cancer, while the Western diet, characterized by higher intakes of fats and processed foods, was positively associated with breast cancer risk. Although we controlled for total daily energy intake, which was calculated based on the frequency of consumption and portion size, we did not adjust for the type of diet or specific foods consumed. Thus, it remains possible that the attenuation in the HRs found among Hispanic women could be attributed to diet. Few studies have examined the effect of specific dietary patterns on breast cancer outcomes. ${ }^{23,30-33}$

We previously reported that there is a nonsignificant increased risk of breast cancer-specific mortality in Hispanic compared to NHW women in this study $(\mathrm{HR}=1.21 ; 95 \% \mathrm{CI}$ 0.84-1.76) that is attenuated with adjustment for age, stage of disease, lymph node status, and ER status. ${ }^{34}$ The focus of the current analysis is on the association of BMI with survival for 
which we find evidence for a potential modification by ethnicity. The question of whether ethnicity modifies the association of BMI with survival is independent of whether survival differs by ethnicity. The absence of a main effect for ethnicity does not preclude the possibility for effect modification between ethnicity and BMI. ${ }^{35}$

Susceptibility to obesity is influenced by genetic variation or interaction of genes with energy intake and/or expenditure (energy balance). ${ }^{2}$ The associations between breast cancer risk and putative obesity-related genes, such as interleukin-6, ERalpha, and androgen receptor, were investigated in the 4Corners Women's Health, and these risks were found to differ between Hispanic and non-Hispanic ethnic groups. ${ }^{36,37}$ The design of this study was closely similar to the NMWHS. Thus, differences between ethnic groups for obesity-associated genes could potentially alter ethnic-specific risks for breast cancer mortality. These genetic factors, for the most part, remain unknown.

A variety of biological mechanisms may underlie the association of obesity and breast-specific cancer mortality. Obesity is associated with insulin resistance and hyperinsulinemia. ${ }^{38}$ Some studies suggest that hyperinsulinemia is positively associated with breast cancer risk as well as later stage at diagnosis and poorer prognosis, ${ }^{39,40}$ including increased breast cancer recurrence and decreased survival. ${ }^{40}$ Obesity is also associated with increased inflammation, angiogenesis, and changes in serum levels of free-estrogen, leptin, and adiponectin. ${ }^{41,42}$ The balance between adiponectin (anti-inflammatory) and leptin (pro-inflammatory) could play an important role in the development and progression of breast cancer. Both adipokines are secreted by adipose tissue and oppose the action of each other. ${ }^{41}$ Obesity is associated with increased serum leptin and reduced adiponectin levels. Preclinical studies have found that high levels of circulating leptin in obese women may function as a growth-enhancing factor; while low levels of adiponectin allow leptin's growthpromoting effects. ${ }^{41}$ The Health, Eating, Activity and Lifestyle (HEAL) study, which includes Hispanic breast cancer survivors in New Mexico, recently reported that serum adiponectin was associated with better breast cancer survival. ${ }^{38}$ However, serum adiponectin levels were significantly higher in NHW than Hispanic women who did not differ significantly for mean BMI. Ethnic differences by these adipokines could not be evaluated because serum specimens were not collected in NMWHS.

It has also been hypothesized that obese women may be more susceptible to undertreatment; however, findings have been mixed. ${ }^{43-45}$ There are concerns that obese women could potentially experience toxic effects if chemotherapy doses were based on their actual body weight. ${ }^{43}$ It also has been suggested that diabetic women may receive less aggressive treatment because of their diabetes-related complications ${ }^{11,46} ; 38 \%$ of the obese breast cancer cases were diabetic.

Strengths of the current study include a population-based study design with a large number of Hispanic women. Our study is one of few epidemiological studies that has investigated the association between obesity and breast cancerspecific mortality among Hispanic and NHW women from the United States. Additionally, extensive lifestyle factor data collected through an in-person interview allowed for adjustment of multiple covariates of interest, including physical activity, comorbidities, and estrogen use. Vital status, cause of death, prognostic markers, and breast cancer treatment history were available through the NDI and NMTR. Vital status statistics through the NDI are documented to have high reliability (sensitivity $\geq 87 \%$ ). ${ }^{47}$

There are several limitations that should be considered. First, the total cohort size is insufficient to provide enough statistical power to declare the interactions of ethnicity with breast cancer-specific and all-cause mortality to be statistically significant at $p<0.05$. Nonetheless, the magnitude of the differences between ethnic groups in the HRs supports potential effect modification. As noted previously, similar disparities between Hispanic and NWH women for breast cancer risk and tumor prognostic markers have been noted in other studies conducted in the southwestern United States. ${ }^{27,28,36}$ Second, BMI was based on self-reported weight and height at time of NMWHS interview. A systematic review comparing direct versus selfreported height and weight found that underreporting of weight and overreporting of height is common in women. ${ }^{48}$ However, findings from a previous study of women diagnosed with breast cancer in New Mexico (1996-1999) based on weight from a variety of sources (measurement at interview, self-report, medical record), reported high correlations between direct and indirect measures, and no differences between Hispanic and NHW women. ${ }^{27}$ Thus, bias due to differential misclassification by ethnicity or obesity does not appear likely. Another limitation is that changes in BMI, which may have occurred after the interview and before death or end of followup, were not accessible for deceased cases. Although previous reports have included the underweight BMI category $\left(<18.5 \mathrm{~kg} / \mathrm{m}^{2}\right)$, in this study there were only 18 cases in this group. Meaningful differences between HRs were not found when models were tested with and without these cases. Finally, some of the stratified analyses were based on small numbers, and findings may not be generalizable.

\section{Conclusions}

The present analysis provides further evidence that obesity is associated with an increased risk for all-cause mortality and to a lesser extent breast cancer-specific mortality in NHW breast cancer cases. However, our results suggest that this association may be attenuated in Hispanic women. The reason for this attenuation remains to be established. The ethnic differences in the associations of obesity with all-cause mortality were similar to those for breast cancer-specific mortality. This finding is not surprising because nearly $60 \%$ of total mortality was attributable to breast cancer in this cohort. In contrast, the association of obesity with non-breast cancer mortality due primarily to cardiovascular disease was closely similar in Hispanic and NHW women. Obesity is just one of the many established risk factors for the development and prognosis of breast cancer; however, it is one of few that can be modified and is thereby considered a preventable risk factor. Public health recommendations to improve survival through weight control following a breast cancer diagnosis should consider disparities between ethnic groups.

\section{Acknowledgments}

This work was supported through grants R01-CA105266 and R01-CA55730 from the National Cancer Institute, the James Graham Brown Cancer Center, and KG090926 from the 
Susan G. Komen, Breast Cancer Disparities Epidemiology Research Training Program.

\section{Author Disclosure Statement}

No competing financial interests exist.

\section{References}

1. Reeves GK, Pirie K, Beral V, et al. Cancer incidence and mortality in relation to body mass index in the Million Women Study: cohort study. BMJ 2007;335:1134.

2. Calle EE, Thun MJ. Obesity and cancer. Oncogene 2004; 23:6365-6378.

3. Parr CL, Batty GD, Lam TH, et al. Body-mass index and cancer mortality in the Asia-Pacific Cohort Studies Collaboration: pooled analyses of 424,519 participants. Lancet Oncol 2010;11:741-752.

4. Abrahamson PE, Gammon MD, Lund MJ, et al. General and abdominal obesity and survival among young women with breast cancer. Cancer Epidemiol Biomarkers Prev 2006;15: 1871-1877.

5. Bessonova L, Marshall SF, Ziogas A, et al. The association of body mass index with mortality in the California Teachers Study. Int J Cancer 2011;129:2492-2501.

6. Carmichael AR, Bendall S, Lockerbie L, et al. Does obesity compromise survival in women with breast cancer? Breast 2004;13:93-96.

7. Chen $X$, Lu W, Zheng W, et al. Obesity and weight change in relation to breast cancer survival. Breast Cancer Res Treat 2010;122:823-833.

8. Thivat E, Therondel S, Lapirot $\mathrm{O}$, et al. Weight change during chemotherapy changes the prognosis in non metastatic breast cancer for the worse. BMC Cancer 2010;10:648.

9. Caan BJ, Kwan ML, Hartzell G, et al. Pre-diagnosis body mass index, post-diagnosis weight change, and prognosis among women with early stage breast cancer. Cancer Causes Control 2008;19:1319-1328.

10. Petrelli JM, Calle EE, Rodriguez C, et al. Body mass index, height, and postmenopausal breast cancer mortality in a prospective cohort of US women. Cancer Causes Control 2002;13:325-332.

11. Conroy SM, Maskarinec G, Wilkens LR, et al. Obesity and breast cancer survival in ethnically diverse postmenopausal women: the Multiethnic Cohort Study. Breast Cancer Res Treat 2011;129:565-574.

12. Lu Y, Ma H, Malone KE, et al. Obesity and survival among black women and white women 35 to 64 years of age at diagnosis with invasive breast cancer. J Clin Oncol 2011; 29:3358-3365.

13. Gilliland FD, Hunt WC, Baumgartner KB, et al. Reproductive risk factors for breast cancer in Hispanic and non-Hispanic white women: the New Mexico Women's Health Study. Am J Epidemiol 1998;148:683-692.

14. Li R, Gilliland FD, Baumgartner K, et al. Hormone replacement therapy and breast carcinoma risk in Hispanic and non-Hispanic women. Cancer 2002;95:960-968.

15. Gilliland FD, Li YF, Baumgartner K, et al. Physical activity and breast cancer risk in Hispanic and non-Hispanic white women. Am J Epidemiol 2001;154:442-450.

16. Baumgartner KB, Annegers JF, McPherson RS, et al. Is alcohol intake associated with breast cancer in Hispanic women? The New Mexico Women's Health Study. Ethn Dis 2002;12:460-469.
17. University of Texas Health Science Center at Houston, School of Public Health. FFDEAP: food frequency data entry and analysis program. Version 1.1. Houston: University of Texas-Houston Health Science Center, 1991.

18. United States Department of Agriculture. United States Department of Agriculture nutrient database for individual intake surveys, Release 7.0. Springfield, VA: National Technical Information Service, 1993.

19. Physical status: the use and interpretation of anthropometry. Report of a WHO Expert Committee. World Health Organ Tech Rep Ser 1995;854:1-452.

20. Ainsworth BE, Haskell WL, Herrmann SD, et al. 2011 Compendium of physical activities: a second update of codes and MET values. Med Sci Sports Exerc 2011;43:1575-1581.

21. Cox DR. Regression models and life tables. JR Stat Soc 1972;34:187-220.

22. Hosmer D, Lemeshow S. Applied logistic regression. New York: Wiley, 1989.

23. Patterson RE, Cadmus LA, Emond JA, et al. Physical activity, diet, adiposity and female breast cancer prognosis: a review of the epidemiologic literature. Maturitas 2010;66:5-15.

24. Protani M, Coory M, Martin JH. Effect of obesity on survival of women with breast cancer: systematic review and metaanalysis. Breast Cancer Res Treat 2010;123:627-635.

25. Ryu SY, Kim CB, Nam CM, et al. Is body mass index the prognostic factor in breast cancer? A meta-analysis. J Korean Med Sci 2001;16:610-614.

26. Maskarinec G, Pagano I, Lurie G, et al. Factors affecting survival among women with breast cancer in Hawaii. J Womens Health (Larchmt). 2011;20:231-237.

27. Baumgartner KB, Hunt WC, Baumgartner RN, et al. Association of body composition and weight history with breast cancer prognostic markers: divergent pattern for Hispanic and nonHispanic white women. Am J Epidemiol 2004; 160:1087-1097.

28. Slattery ML, Sweeney C, Edwards S, et al. Body size, weight change, fat distribution and breast cancer risk in Hispanic and non-Hispanic white women. Breast Cancer Res Treat 2007;102:85-101.

29. Murtaugh MA, Sweeney C, Giuliano AR, et al. Diet patterns and breast cancer risk in Hispanic and non-Hispanic white women: the Four-Corners Breast Cancer Study. Am J Clin Nutr 2008;87:978-984.

30. Hauner H, Hauner D. The impact of nutrition on the development and prognosis of breast cancer. Breast Care (Basel) 2010;5:377-381.

31. Rock CL. Diet and breast cancer: can dietary factors influence survival? J Mammary Gland Biol Neoplasia 2003;8:119-132.

32. Kwan ML, Weltzien E, Kushi LH, et al. Dietary patterns and breast cancer recurrence and survival among women with early-stage breast cancer. J Clinical Oncol 2009;27:919-926.

33. Kroenke $\mathrm{CH}$, Fung TT, Hu FB, et al. Dietary patterns and survival after breast cancer diagnosis. J Clinical Oncol 2005;23:9295-9303.

34. Baumgartner KB, Baumgartner RN, Denkhoff S, et al. Differences in long-term survival for Hispanic and nonHispanic white women with breast cancer. Cancer Res 2011; 71(24 Suppl):Abstract nr S1-7.

35. Szklo M, Nieto J. Defining and assessing heterogeneity of effects: interaction. In: Epidemiology: beyond the basics. Sudbury, MA: Jones and Bartlett Publishers, Inc., 2007:183-226.

36. Slattery ML, Sweeney C, Herrick J, et al. ESR1, AR, body size, and breast cancer risk in Hispanic and non-Hispanic white women living in the southwestern United States. Breast Cancer Res Treat 2007;105:327-335. 
37. Slattery ML, Curtin K, Sweeney C, et al. Modifying effects of IL-6 polymorphisms on body size-associated breast cancer risk. Obesity (Silver Spring) 2008;16:339-347.

38. Duggan C, Irwin ML, Xiao L, et al. Associations of insulin resistance and adiponectin with mortality in women with breast cancer. J Clin Oncol 2011;29:32-39.

39. Goodwin PJ, Ennis M, Bahl M, et al. High insulin levels in newly diagnosed breast cancer patients reflect underlying insulin resistance and are associated with components of the insulin resistance syndrome. Breast Cancer Res Treat 2009;114:517-525.

40. Goodwin PJ, Ennis M, Pritchard KI, et al. Fasting insulin and outcome in early-stage breast cancer: results of a prospective cohort study. J Clin Oncol 2002;20:42-51.

41. Grossmann ME, Ray A, Nkhata KJ, et al. Obesity and breast cancer: status of leptin and adiponectin in pathological processes. Cancer Metastasis Rev 2010;29:641-653.

42. Fontana L, Eagon JC, Trujillo ME, et al. Visceral fat adipokine secretion is associated with systemic inflammation in obese humans. Diabetes 2007;56:1010-1013.

43. Griggs JJ, Sorbero ME, Lyman GH. Undertreatment of obese women receiving breast cancer chemotherapy. Arch Intern Med 2005;165:1267-1273.

44. Brewster AM, Etzel C, Zhou R, et al. The impact of obesity on receipt of adjuvant chemotherapy for breast cancer in the National Comprehensive Cancer Network (NCCN) centers. Breast Cancer Res Treat. 2011;130:897-904.
45. Buist DS, Ichikawa L, Prout MN, et al. Receipt of appropriate primary breast cancer therapy and adjuvant therapy are not associated with obesity in older women with access to health care. J Clin Oncol 2007;25:3428-3436.

46. Srokowski TP, Fang S, Hortobagyi GN, et al. Impact of diabetes mellitus on complications and outcomes of adjuvant chemotherapy in older patients with breast cancer. J Clin Oncol 2009;27:2170-2176.

47. Cowper DC, Kubal JD, Maynard C, et al. A primer and comparative review of major US mortality databases. Ann Epidemiol 2002;12:462-468.

48. Gorber SC, Tremblay M, Moher D, et al. A comparison of direct vs. self-report measures for assessing height, weight and body mass index: a systematic review. Obes Rev 2007; 8:307-326.

Address correspondence to: Avonne Connor, PhD University of Louisville School of Public Health and Information Sciences Department of Epidemiology and Population Health

485 E. Gray Street

Louisville, KY 40202

E-mail: aeconn02@louisville.edu 